American Journal of Applied Sciences 4 (12): 1071-1074, 2007

ISSN 1546-9239

(C) 2007 Science Publications

\title{
Effect of Internal Flow Patterns on Heat transfer Performance of a Closed-end Oscillating Heat Pipe at Vertical Position
}

\author{
${ }^{1}$ S. Rittidech, ${ }^{1}$ L. Yodluk and ${ }^{2} \mathrm{~K}$. Songsorn \\ ${ }^{1}$ Faculty of Engineering, Mahasarakham University, 41/20 Khamriang Sub-District, \\ Kantartawichai District, Mahasarakham, 4450,Thailand \\ ${ }^{2}$ Faculty of Engineering, Ubonrarjathanee University, Ubonrarjathanee, 34910
}

\begin{abstract}
This article describes the effects of varying bond numbers (Bo) and aspect ratios (Le/d) on flow pattern of a close-end oscillating heat-pipe (CEOHP). The CEOHPs used employed a glass tube with inside diameter of $3 \mathrm{~mm}$. The length of evaporator of 100 and $150 \mathrm{~mm}$ (the lengths of evaporator adiabatic and condenser section were equal) were employed with 10 turns. Observation of the flow pattern was conducted at $90^{\circ}$ from the horizontal plane, with bond numbers of 1.8 and 2.6 with aspect ratios of 33.3 and 50. R $141 \mathrm{~b}$ and ethanol were used as the working fluids with filling ratio of $50 \%$ of internal volume of tube. The evaporator section was heated by heater and the condenser section was cooled by distilled water. The working temperature was controlled at $50^{\circ} \mathrm{C}$. The temperature at adiabatic section and the inlet-outlet of distilled water at condenser section were recorded. The photographs of flow patterns at specific times were recorded at evaporator section by a digital camera and video camera were used to observe the flow patterns at evaporator section condenser together with adiabatic section and total part of CEOHP. It was found that the maximum heat flux occurred with Le/d 33 and Bo 2.6 an inside phenomena occurred is a dispersed bubble flow and slug flow.
\end{abstract}

Key words: Flow patterns, Closed-end oscillating heat-pipe Bond number, Aspect ratio

\section{INTRODUCTION}

The rapid development of practical engineering application for micro-devices, micro-systems, advanced material designs and manufacturing (including compact heat exchangers and devices for cooling electronic equipment). These heat transfer device for high heat load, conventional heat pipes have several limitation, such as the capillary limit when the diameter of the pipe is comparatively small, and the entrainment limit as the temperature difference is high. This problem becomes especially crucial when using heat pipe to cool electronic devices. Closed-end oscillating heat pipe (CEOHP) in figure 1 is effective heat transfer device. The CEOHP its properties of transferring heat in any orientation, its quicker response. In quantitative determining for the heat transfer characteristics of the CEOHP at the normal operating condition. Rittidech et al. $^{[2]}$ have the conducted experiments with fill ratio 50\% and working temperature of the $50^{\circ} \mathrm{C}$. The variable parameters were the inclination angle, inner diameter of the tube, number of turn, working fluid and evaporator section length. It was found from the experimental results that when the inner diameter increased the heat flux increased. As the evaporator section length increased when latent heat of the vaporization of working fluid increased. The number of turns affected the heat flux such that the maximum heat flux is occurred 14 turns. However, when the number of turns was increased or decreased. Therefore, the effect of number of turns on heat flux was not made clear. Since that time, many studies on this device have been conducted. Among all the analysis and the experiment of the internal flow patterns are the major problems of the research, because they are crucial means to under stand the mechanism of the heat transfer in CEOHP. A heat transfer characteristic of the CEOHP is directly affected by internal flow patterns. There are quite a number of research papers about flow behavior of the CEOHP. Gi et al $^{[1]}$ have conducted experiment with R142b by varying the fill ratios and inclination angles. A CEOHP with an ID of $2 \mathrm{~mm}$ and a total length of $8020 \mathrm{~mm}$ was employed, with 10 turns and working temperature of $45^{\circ} \mathrm{C}$ they observed that the working could not circulate thoroughly. The heat transferred by driving force due to the oscillation between heating and cooling section. The internal oscillation occurred at smaller inclination angle and smaller fill ratio. Rittidech et al. ${ }^{[3]}$ investigated the effect of evaporator section lengths number of turn and working fluids on internal flow patterns of a CEOHP at normal operating condition. The CEOHPs used employed a glass tube with inside diameter of $2.03 \mathrm{~mm}$. The lengths of evaporator, adiabatic (which is equal to

Corresponding Author: S. Rittidech, Faculty of Engineering, Mahasarakham University, Khamriang Campus, Kantharawichai, Mahasarakham, 44150, Thailand 
condenser section) were $50 \mathrm{~mm}$ with 15 turns. The inclination angle is $90^{\circ}$ from horizontal plane. R $141 \mathrm{~b}$ was us as the working fluid with filling ratio of $50 \%$ of internal volume of tube. The evaporator section was heating plate and the condenser section was cooled by distilled water. It was found from the experiment that, as the evaporator section length decreases from 150 to $50 \mathrm{~mm}$ the main flow changes from the slug flow with annular flow to slug flow with bubble flow. Effect of number of turns can not be clearly identified. If the working fluid change from R141b to R123, the main flow change from the slug flow with annular flow to slug flow with bubble flow. They are concerned with heat transfer characteristics and flow visualization effect of working fluid evaporator section length number of turns and the operation mechanism of CEOHP cannot be clearly identified. Therefore, is causing an increased interest by the heat transfer community into research in the transport phenomena in CEOHP. Their results of flow pattern were clear and informative, but no results were recorded to Bond number (Bo) and aspect ratio (ratio of evaporator section length to inner diameter, Le/d). Since there are two main phenomena occurring inside a CEOHP, i.e., liquid slug and vapor slug counter-current flow phenomenon and boiling phenomenon. Bo represents the state of vapor slug which occurs in nucleate boiling. Since Bo shows the relationship between buoyancy force and surface tension force of the slug and Le/d indicates the size of a CEOHP. It is, therefore, the objective of this article to investigate the effects of Bond number and aspect ratio on internal flow patterns of a CEOHP at normal operating conditions.

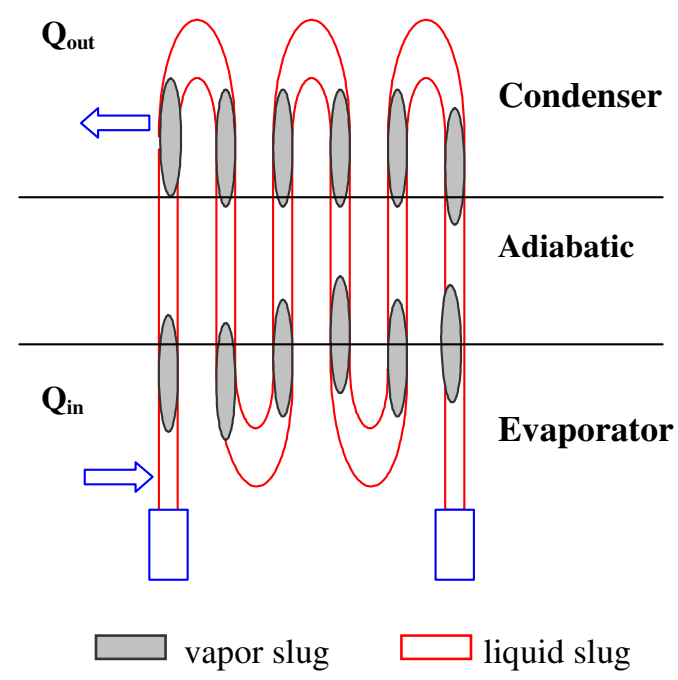

Fig.1: Closed-end oscillating heat pipe (CEOHP).

\section{MATERIALS AND METHODS}

Figure 2 shows an experimental setup which consists of a CEOHP with the lengths of evaporator, adiabatic (which is equal to condenser sections) were 100 and $150 \mathrm{~mm}$ respectively. The selected CEOHPs were made of the Pyrex glass tube with the internal diameter of $3 \mathrm{~mm}$. The evaporator glass section was heated by heater. The condenser section was cooled by distilled water, which was circulated from the cold bath (EYELA CA-1111, volume 6.01 with an operating temperature range of -20 to $30^{\circ} \mathrm{C}$ and $\pm 2{ }^{\circ} \mathrm{C}$ accuracy) was used to pump the water into the cooling jacket. The mass flow rate inside the cooling jacket was measured by floating rotameter (Platon PTF2 ASS-C with a measure flow rate of $0.2 \mathrm{~L} / \mathrm{min}$ to $1.5 \mathrm{~L} / \mathrm{min}$ ) and 4 points of thermocouples (OMEGA type K) were installed at the inlet and outlet of condenser section to determine the heat transfer rate. The temperature probes were installed 4 points at high temperature aluminum plate of evaporator and 1 point for ambient to determine the heat loss. The temperature recorder (Yokogawa DX 200 with $\pm 0.1^{\circ} \mathrm{C}$ accuracy, 20 channel input and $200^{\circ} \mathrm{C}$ to $1100^{\circ} \mathrm{C}$ measurement temperature range) was used with type $\mathrm{K}$ thermocouples (Omega with $\pm 1^{\circ} \mathrm{C}$ accuracy) was uses to monitor all temperatures at specified times. Moreover, 2 point of thermocouple were installed at the middle position. During the experiment the angle was set at 90 degrees from horizontal plane. Video camera (Sony CCD-TR618E) was employed to continuously record the flow patterns at the evaporator section, condenser together with adiabatic section, and total part of CEOHPs. The digital camera (DSC-S75) was used to record flow patterns of evaporator section at specified times. The scale was attached to the apparatus to measure the length and velocity of vapor bubble. The controlled parameters were: Tube internal diameter of $3.00 \mathrm{~mm}$, working temperature of $50^{\circ} \mathrm{C}$, filling ratio of $50 \%$ (by total volume). The variable parameters were: Le/d 33 and 50 (to observed the effect of Le/d). Bo 1.8 and 2.6. The experiment was conducted as follows; Firstly, the CEOHP was with the working and set into he test rig. The temperature of the heater and cold baths were set as the required value, and cold fluids were supplied to the jackets of both and the condenser section. After a steady state was reached, continuous movie were recorded by video cameras while photographs were taken at specified times by digital camera. In the mean time, temperature and heat transfer rate were monitored. Then the Bo and Le/d were varied according to the required conditions.

\section{RESULS AND DISCUSSION}

Visualization at the evaporator section was concentrated since the major phenomena occurred in the part, the total flow, which cannot be presented in the paper could, however, be observed by video movie. The evaporator section experiment results will be referred to 
this research. The vapor bubble length was measured as the length of the two ends of vapor at specified time. The internal flow patterns at specific aspect ratios have been presented at for all bond number. It can be concluded from the experimental results as follows;

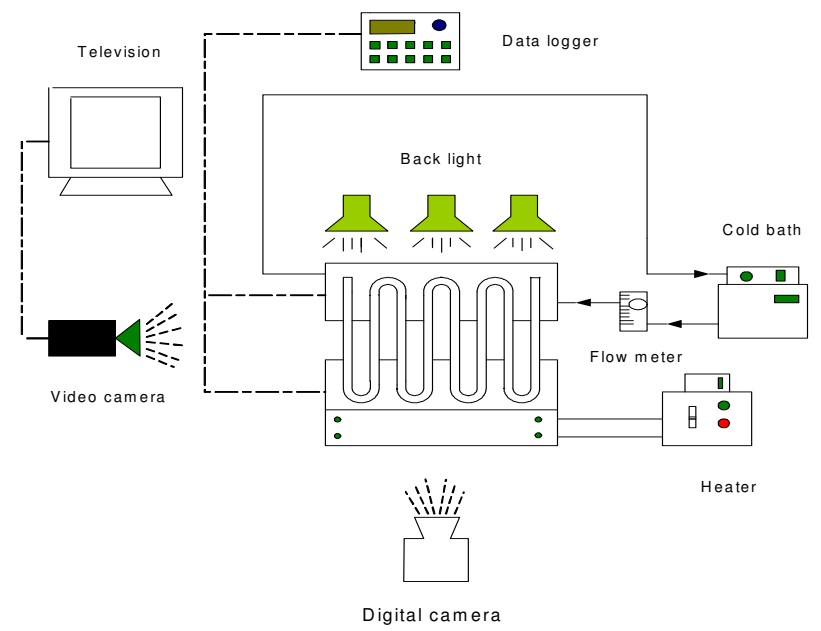

Fig. 2: Experimental set-up

\section{Flow patterns of the aspect ratio 33.3}

At Bond number of 1.8: Figure 3 shows the inside flow patterns of the CEOHP with a Le/d of 33.3 and Bo 1.8 at the vertical position. It can be observe with a heat flux of $0.43 \mathrm{~kW} / \mathrm{m}^{2}$, the slug flow with more nucleation sites can be observe in the lower part of the evaporator. The annular flows dominate in the middle and upper parts of evaporator. The length of the annular slug is approximately $0.082 \mathrm{~m}$. The velocity of vapor slug is $0.10 \mathrm{~m} / \mathrm{s}$. It can be stated that patterns of the annular flows dominate at an aspect ratio of 33.3 and Bo 1.8 at a $90^{\circ}$ angle.

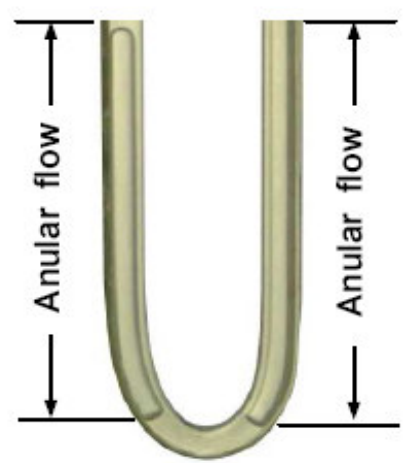

Fig. 3: Le/d 33,Bo = 1.8

At Bond number of 2.6: Figure 4 shows the inside flow patterns of the CEOHP with a Le/d of 33.3 and Bo 2.6 at the vertical position. It can be observe with a heat flux of $1.56 \mathrm{~kW} / \mathrm{m}^{2}$, bubble flow with more nucleation sites can be observe in the lower part of the evaporator. While in the middle and upper parts, the length of bubble and dispersed bubble flow is approximately $0.021 \mathrm{~m}$. The velocity of vapor slug is $0.25 \mathrm{~m} / \mathrm{s}$. It can be stated that patterns of the small slug and dispersed bubble flows dominate at an aspect ratio of 33.3 and Bo 2.6 at a $90^{\circ}$ angle.

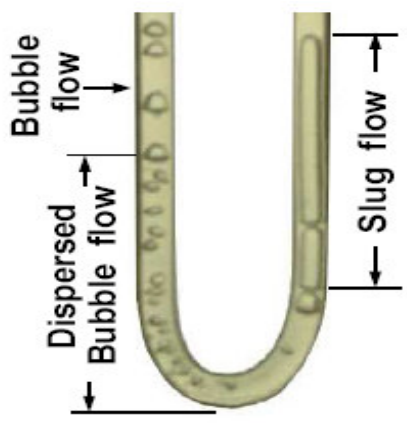

Fig. 4: Le/d 33,Bo = 2.6

\section{Flow patterns of the aspect ratio 50}

At Bond number of 1.8: Figure 5 shows the inside flow patterns of the CEOHP with a Le/d of 50 and Bo 1.8 at the vertical position. It can be observe with a heat flux of $0.87 \mathrm{~kW} / \mathrm{m}^{2}$, slug flow with more nucleation sites can be observe in the lower part of the evaporator. While slug and annular flows dominate in the middle and upper parts of evaporator. The length of the vapor slug is approximately $0.062 \mathrm{~m}$. The velocity of vapor slug is $0.15 \mathrm{~m} / \mathrm{s}$. It can be stated that patterns of the slug and annular flow dominate at an aspect ratio of 50 and Bo 1.8 at a $90^{\circ}$ angle.

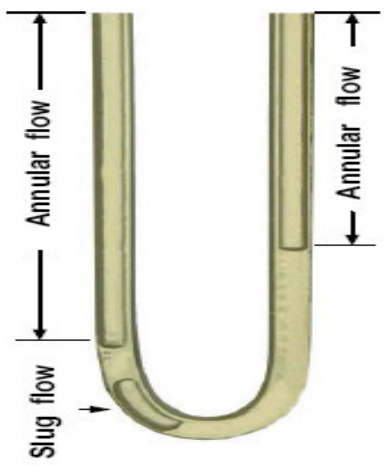

Fig.5: Le/d 50,Bo=1.8

At Bond number of 2.6: Figure 6 shows the inside flow patterns of the CEOHP with a Le/d of 50 and Bo 2.6 at the vertical position. It can be observe with a heat 
flux of $0.89 \mathrm{~kW} / \mathrm{m}^{2}$, slug flow with more nucleation sites can be observe in the lower part of the evaporator, slug and annular flows dominate in the middle and upper parts of evaporator. The length of the vapor slug is approximately $0.051 \mathrm{~m}$. The velocity of vapor slug is $0.170 \mathrm{~m} / \mathrm{s}$. It can be stated that patterns of the slug and annular flow dominate at an aspect ratio of 50 and Bo 2.6 at a $90^{\circ}$ angle.



Fig. 6. Le/d 50,Bo = 2.6

From these studies, it can be explained that the heat can be transferred by the following mechanism: as heat is supplied at the evaporator, the vapor bubbles originate in the evaporator section and latent heat is releases. It can be conclude that, the maximum heat flux occurred with dispersed bubble flow and small slug flow, that shown in Fig. 4. Because when the Le/d is short the bubbles can move to the condenser at high speed and then quick releases heat in this section.

\section{CONCLUSION}

From visual study of the effects of bond number and aspect ratio on the internal flow patterns of a closeend oscillating heat pipe at normal operating conditions, it can be concluded that the inside phenomena slug flow is small occurred high heat flux.

\section{ACKNOWLEDGEMENTS}

The research has been supported generously by the Faculty of Engineering Mahasarakham University. The authors express their sincere appreciation for all of support provided.

\section{REFERENCES}

1. Gi K., Sato F., and Maesawa S., 1999. Flow visualization experimental on oscillating heat pipe, in Proceeding of the $11^{\text {th }}$ International Heat Pipe Conference, Musashinoshi Tokyo, Japan, pp.149153

2. Rittidech S., Terdtoon P., Murakami M. Kamonpet P., and Jompakdee W., 2000. Effect of inclination angle, evaporator lengths and working fluid on heat transfer characteristics of closed-end oscillating heat pipe, In proceeding of the six international heat pipe symposium, Chiang Mai Thailand, pp. 413-421

3. Rittidech S., Terdtoon P., Murakami M., Kamonpet P., and Jompakdee W., 2003. Effect of evaporator section lengths number of turns and working fluid on internal flow patterns of a closed-end oscillating heat pipe, In proceeding of the seven international heat pipe symposium, Jeju, Korea, pp. 217-223. 